\title{
LA SUISSE EN PANNE DE POLITIQUES DE FIN DE CARRIÈRE EN ENTREPRISE
}

\author{
Kabak O Jean-Pascal Corréa, René Knüsel, Jean-Marie Le Goff
}

Caisse nationale d'assurance vieillesse | « Retraite et société »

2017/2 $\mathrm{N}^{\circ} 77$ | pages 17 à 44

ISSN $1167-4687$

ISBN 9782858231096

Article disponible en ligne à l'adresse :

https://www.cairn.info/revue-retraite-et-societe-2017-2-page-17.htm

\section{Pour citer cet article :}

Kabak O Jean-Pascal Corréa et al., « La Suisse en panne de politiques de fin de carrière en entreprise », Retraite et société 2017/2 ( $\left.\mathrm{N}^{\circ} 77\right)$, p. 17-44.

Distribution électronique Cairn.info pour Caisse nationale d'assurance vieillesse.

(C) Caisse nationale d'assurance vieillesse. Tous droits réservés pour tous pays.

La reproduction ou représentation de cet article, notamment par photocopie, n'est autorisée que dans les limites des conditions générales d'utilisation du site ou, le cas échéant, des conditions générales de la licence souscrite par votre établissement. Toute autre reproduction ou représentation, en tout ou partie, sous quelque forme et de quelque manière que ce soit, est interdite sauf accord préalable et écrit de l'éditeur, en dehors des cas prévus par la législation en vigueur en France. Il est précisé que son stockage dans une base de données est également interdit. 
La Suisse

en panne de politiques

de fin de carrière

en entreprise

Kabak 0 Jean-Pascal Corréa

Assistant-doctorant

René Knüsel

Professeur

et Jean-Marie Le Goff

Maître d'enseignement et de recherche

Institut des sciences sociales de l'université de Lausanne 




Corréa K. O J.-P., Knüsel R., Le Goff J.-M., 2017, « La Suisse en panne de politiques de fin de carrière en entreprise $»$, Retraite et société, n ${ }^{\circ} 77$, p. 18-44.

Si le niveau de qualification comme l'actualisation des compétences jouent un rôle important dans le maintien ou le retour en emploi des travailleurs âgés, les dispositions comme l'attitude adoptée par les employeurs peuvent aussi contribuer à circonscrire les risques de précarité d'emploi en fin de carrière. La Suisse, caractérisée par un marché du travail de type libéral, se singularise par une quasi-absence de politiques et de pratiques favorables à la (ré)intégration des travailleurs de plus de 50 ans, rendant ainsi les chômeurs âgés très vulnérables. À quelques exceptions près, les efforts déployés par ces personnes en vue d'un retour en emploi sont contrecarrés par un marché de l'emploi exigeant. En conséquence, les hommes et les femmes en fin de carrière voient leurs perspectives d'un retour à l'emploi s'éloigner. En comparaison européenne, le risque pour un travailleur âgé de connaître le chômage est moindre en Suisse, mais celui qui connaît pareille situation a une chance nettement plus réduite de retrouver un emploi. Une spirale négative qui se traduit par un affaiblissement de la situation matérielle de ces sans-emploi une fois à la retraite tend à se mettre en place. Il apparaît clairement que le risque de précarité en fin de carrière est encore plus accentué pour les femmes, puisque l'emploi tout comme les prestations de la plupart des assurances ont été pensés avec pour référence un homme salarié à temps plein.

Mots-clés : travailleurs âgés ; chômage ; genre ; précarité ; politiques publiques de vieillesse ; employeurs ; discrimination

\section{Switzerland comes up short on late-career management policies at businesses}

While qualifications and upskilling play an important role for seniors in maintaining and returning to employment, the systems and attitudes of employers can also serve to limit the risks of occupational precarity in late careers. With its liberal job market, Switzerland is distinguished by a near total lack of policies and practices favourable to the (re)integration of workers aged over 50, making unemployed seniors highly vulnerable. With very few exceptions, the efforts made by this population to return to employment are thwarted by a highly demanding labour market. As a result, men and women at the latter stages of their careers are finding it increasingly difficult to return to employment. Compared with Europe, the risk of unemployment for senior workers is lower in Switzerland, but those who are unemployed have a considerably lower chance of finding a job. A negative spiral tends to develop, reflected in a decline in the material well-being of unemployed seniors in retirement. The risk of late-career precarity is clearly higher for women, as employment and most pension insurance schemes are designed with a fulltime salaried male worker in mind.

Keywords: senior workers; unemployment; gender; precarity; public policies on ageing; employers; discrimination 
'émergence de la question des travailleurs âgés et de leurs difficultés sur le marché du travail est plus récente en Suisse que chez ses voisins européens, notamment en France ou en Allemagne, où ces difficultés étaient apparues dès la fin des années 1970 (Guillemard, 1993). En Suisse, elles se signalent à l'occasion de la crise économique du début des années 1990 (Le Goff, 1999). Par ailleurs, c'est aussi lors de ces années que des instances internationales (en particulier l'Organisation de coopération et de développement économiques - $\mathrm{OCDE}$ ) commencent à mettre en garde les pays développés contre les effets du vieillissement de la population et leurs conséquences sur les régimes de retraite et à inciter les pouvoirs publics de ces pays à mettre en place des politiques visant à prolonger la vie active. Dans le cas de la France ou de l'Allemagne, ces mises en garde prenaient à contresens les politiques développées jusqu'alors, puisqu'elles favorisaient des mesures permettant aux travailleurs âgés de sortir tôt du marché du travail (politiques de retraites anticipées, de sorties par le chômage ou l'invalidité).

Dans le cas de la Suisse, en revanche, la contradiction entre les difficultés observées des travailleurs âgés à se maintenir sur le marché du travail et une volonté politique à ne pas favoriser les départs précoces du marché du travail a été d'emblée mise en évidence. Cette volonté politique n'est toutefois pas synonyme d'une instauration de politiques visant à protéger les travailleurs âgés. À l'absence d'une protection législative plus importante des travailleurs, spécialement contre les discriminations face à l'emploi, s'ajoute un renvoi à la responsabilité individuelle de tout entreprendre pour trouver ou retrouver un emploi.

De leur côté, les associations d'employeurs multiplient les prises de position insistant sur l'importance de maintenir en emploi les plus âgés. Néanmoins, en dépit des discours, peu d'actions sont entreprises en ce sens. En outre, les termes de travailleurs âgés ou de seniors, tels qu'utilisés dans ces discours, dessinent une catégorie générique qui ne permet pas de distinguer les statuts sociaux ou les parcours professionnels antérieurs. Ainsi, les spécificités des femmes âgées de 50 ans ou plus, qui appartiennent à des générations ayant encore souvent interrompu leur activité professionnelle au moment de la naissance de leur enfant, avant de retourner sur le marché du travail pour occuper des emplois à temps partiel, ne sont pas prises en considération.

De cette absence de politique résulte une sorte de marais barométrique dans lequel les travailleurs âgés sont livrés à leur sort, au gré de la conjoncture. L'objectif dans cet article 
est de mieux comprendre pourquoi et comment les travailleurs âgés, et plus particulièrement les femmes, sont mis en difficulté soit par la quasi-absence de politiques spécifiques des entreprises en matière de maintien en emploi, soit par des pratiques et des procédés qui, loin de stimuler la réintégration des demandeurs d'emploi de « 50 ans et plus », ont pour effet de limiter durablement l'accès de cette population au marché du travail.

Les résultats présentés ici s'appuient sur une recherche en cours intitulée « Fin de carrière professionnelle : enjeux suisses d'une situation en voie d'ébullition ${ }^{1} \gg$. Cette recherche vise à mieux comprendre la situation des travailleurs âgés, ainsi que les complémentarités et les contradictions entre les différents acteurs institutionnels (employeurs, responsables d'assurances sociales, etc.) jouant un rôle dans l'insertion ou l'exclusion des travailleurs âgés. Dans le cadre de cette recherche, une série d'enquêtes et d'entretiens a été menée auprès de ces différents acteurs.

Dans le cas présent, nous nous focaliserons plus particulièrement sur les pratiques des employeurs. Au plan théorique, nous partons d'un dénominateur commun - l'absence de politiques de protection des travailleurs âgés - pour identifier les pratiques des employeurs qui maintiennent les seniors dans la précarité d'emploi. Nous ne postulons pas que ces usages traduisent une volonté manifeste de limiter l'accès des seniors à l'emploi. Il s'agit plutôt, en identifiant ici certaines de ces pratiques, de démontrer leurs effets sur l'insertion ou la non-insertion sur le marché du travail des « 50 ans et plus », en particulier les demandeurs d'emploi en fin de carrière professionnelle.

Nous présenterons d'abord les conditions générales, sur le plan législatif en Suisse, qui se caractérisent, d'un côté, par l'absence de protection spécifique du travailleur âgé et, de l'autre, par les conditions exigeantes d'accès au système de protection sociale permettant une retraite sereine. Les approfondissements qui suivront entendent insister sur le positionnement des employeurs, principaux soutiens et bénéficiaires d'une approche peu réglementée du marché de l'emploi qui rend certaines fins de parcours professionnels difficiles à vivre pour un nombre grandissant de salariés.

\section{Spécificités et paradoxes de la Suisse}

\section{Un marché du travail très libéral}

Parmi les pays développés, la Suisse se caractérise par un marché de l'emploi très libéral dans son organisation et son fonctionnement. Ce marché connaît relativement peu de contraintes, en particulier dans l'embauche et le licenciement du personnel. Si les discriminations liées au genre sont prohibées, il n'existe pas en revanche d'interdiction fondée sur une mention d'âge limite, en dépit de l'article 8 de la Constitution fédérale indiquant que « Nul ne doit subir de discrimination du fait notamment de son origine, de sa race, de son sexe, de son âge [...] ». Il est vrai que cette disposition ne contraint que les pouvoirs publics, en tant qu'employeurs. Contrairement à d'autres États libéraux comme les États-Unis ${ }^{2}$ ou des systèmes plus interventionnistes comme ceux de la

\footnotetext{
1. Recherche financée par le Fonds national suisse de la recherche scientifique (FNS), requête $\mathrm{n}^{\circ} 100017149575$.

2. The Age Discrimination in Employment Act of 1967.
} 
plupart des membres de l'Union européenne, les lois régissant les rapports de travail en Suisse ne contiennent aucune mention interdisant l'indication d'un âge maximal. Il est d'ailleurs admis que les institutions publiques mentionnent une limite d'âge si une telle utilité peut se justifier. En conséquence, de nombreuses offres d'emploi mentionnent un « âge idéal » ou un « âge souhaité ». Une enquête menée en 2015 auprès du plus grand portail de l'emploi de Suisse (Job.ch), montrait que moins d'un pour cent des offres parues s'adressaient directement à des candidats âgés de 45 à 65 ans $^{3}$.

Une autre recherche concernant la spécification de l'âge dans les offres d'emploi au cours des dix dernières années indique pourtant que la fréquence de ces mentions tend à diminuer (Buchs, 2016). Alors qu'en 2006, environ $16 \%$ des annonces mentionnaient un âge, elles n'étaient plus que $6 \%$ en 2015, selon les auteurs de cette étude. Une diminution du nombre de discriminations explicites ne signifie cependant pas l'inexistence de pratiques ségrégatives plus en aval dans le processus de recrutement, notamment lors de la sélection des candidats. L'étude relève quelques-unes des manières de faire plus subtiles et indirectes qui se répandent avec des libellés tels que : « Pour compléter équipe jeune et dynamique (...) nous recherchons (...). »

Au plan politique, l'interdiction de ces pratiques n'est pas à l'ordre du jour. Ainsi, à l'issue de la deuxième conférence nationale consacrée aux travailleurs âgés, le conseiller fédéral (rang de ministre) responsable du dossier déclarait ${ }^{4}:$ « Nous avons par ailleurs décidé, lors de la conférence, que les employeurs publics et privés renonceraient à indiquer un âge dans les offres d'emploi lorsque c'est possible. Je m'oppose en revanche à toute intervention exagérée en la matière. Ainsi, l'introduction d'une protection renforcée contre le licenciement à partir d' un certain âge serait contreproductive, car elle réduirait massivement les chances d'embauche des seniors. Le système libéral reposant sur un partenariat social qui a fait ses preuves est un des atouts de la Suisse. Il serait inconsidéré de le compromettre ${ }^{5}$. »

\section{Activité et chômage des travailleurs âgés}

Développées depuis 1991, les enquêtes suisses sur la population active (ESPA) ${ }^{6}$, dont les définitions reposent sur les catégories d'activité, d'emploi et de chômage telles qu'elles ont été définies par le Bureau international du travail (BIT), permettent d'évaluer la situation des travailleurs âgés en Suisse. Ainsi, le taux d'activité des hommes en fin de carrière (55-64 ans) est élevé et stable, se situant entre 75 et $79 \%$ depuis 1995 , ce qui en fait l'un des taux d'activité des plus élevés d'Europe. Durant cette période, les femmes ont vu leur taux d'activité augmenter de $20 \%$ en passant de $46 \%$ en 1995 à $66 \%$ en 2015. Plusieurs raisons sont à l'origine de cette augmentation. Mentionnons l'élévation de l'âge légal de la retraite de 62 ans à 64 ans chez les femmes entre la fin des années 1990 et le début des années 2000. L'augmentation du taux d'activité des femmes seniors

3. Enquête commanditée par le Tages-Anzeiger auprès de Jobs.ch et parue dans les colonnes du journal le 5 février 2015. Une enquête similaire a été menée en Suisse romande, confirmant l'étendue de cette pratique, voir à ce propos Générations (Discriminations... 1.01.2017).

4. Malgré l'opposition initiale du Conseil fédéral, le parlement avait adopté le postulat Rechsteiner chargeant le Conseil fédéral de « convoquer une conférence nationale sur le thème des travailleurs âgés ». Une première conférence a été organisée, en avril 2015, suivie d'une seconde, en avril 2016.

5. Propos du conseiller fédéral, Johann Schneider-Amman, rapportés dans $L a$ Vie économique, $\mathrm{n}^{\circ} 7$, 2015 , p. 58.

6. Équivalent des enquêtes emploi de l'Insee ou des enquêtes sur les forces de travail d'Eurostat (EFT). 
est néanmoins surtout due à un effet de génération. Parmi les générations nées avant la Seconde Guerre mondiale se trouvaient beaucoup de femmes qui arrêtaient de travailler définitivement au moment de la naissance de leur premier enfant. En revanche, les femmes des générations suivantes ont eu pour pratique de retourner sur le marché du travail une fois leurs enfants scolarisés. Ce phénomène s'est accentué pour les générations les plus récentes : certaines femmes choisissant seulement de diminuer leur temps de travail (Diserens, Briant, 1996 ; Widmer, Ritschard, 2009).

Les modalités du chômage des travailleurs âgés sont semblables à celles observées dans de nombreux pays européens, mais dans un contexte où, globalement, le taux est peu élevé. Chez les seniors, il est inférieur à la moyenne des autres groupes d'âge. Ainsi, en 2015, il se situait aux environs de $3 \%$, soit moins que les catégories plus jeunes (25-54 ans) qui comptaient un taux de chômage de plus de 4 \%. Les risques de perte d'emploi sont réduits pour les seniors, tant en ce qui concerne les licenciements - en particulier, dans les branches fortement encadrées par des conventions collectives de travail (CCT) - que les fins de contrats à durée déterminée (CDD). Le chômage intervient plus souvent, en revanche, suite à un accident, une maladie ou une entrée en invalidité$^{7}$ et lorsqu'il concerne les travailleurs âgés, il a pour caractéristique d'être souvent de longue durée. Ainsi, en 2015, la part des chômeurs depuis plus de 12 mois était de 54 \% chez les 55-65 ans contre, respectivement, $45 \%$ et $31 \%$ chez les $40-54$ et $25-39$ ans. Le taux total des chômeurs de longue durée atteignait 1,7 \% chez les actifs de 55 à 64 ans, alors qu'il était de 1,4\% pour l'ensemble de toutes les autres catégories confondues. La situation des chômeurs âgés s'est par ailleurs constamment aggravée depuis $1990^{8}$. La durée de recherche d'emploi des plus de 50 ans est environ une fois et demie plus longue que la moyenne, et deux fois plus longue que chez les demandeurs d'emploi âgés de 15 à 24 ans. En 2015, les « 50 ans et plus » constituaient $43 \%$ de l'ensemble des chômeurs de longue durée. En résumé, les seniors suisses sont donc moins exposés aux risques du chômage que les autres groupes d'âge, mais le temps passé à l'assurance-chômage est plus long que celui des autres catégories d'âge. Il s'apparente ainsi à un chômage d'exclusion.

Un regard sur les seniors sans emploi et n'en cherchant pas permet d'appréhender le processus de leur exclusion. Ainsi, plus d'un quart d'entre eux indiquaient une invalidité permanente ou une incapacité temporaire (Secrétariat d'État à l'économie [Seco], 2016). Une très grande majorité de femmes indiquait des motifs personnels ; une partie d'entre elles n'exerçait pas d'activité professionnelle auparavant.

La situation des travailleurs âgés en Suisse pourrait ainsi paraître moins préoccupante que dans d'autres pays européens. Cependant, le chômage de longue durée et plus généralement, les fortes difficultés rencontrées par les travailleurs âgés à retrouver un emploi contrastent avec l'approche libérale du marché qui prédomine dans ce pays. Si les responsables politiques déduisent qu'un renforcement de la protection des seniors serait contre-productif, une telle affirmation doit être relativisée dans la mesure où une partie des seniors renonce tout simplement à retourner sur le marché de l'emploi, découragée par le contexte ainsi que l'expérience difficile, directe ou indirecte, à se maintenir

7. Op. cit., p. 56 .

8. OCDE, 2014. 
sur le marché du travail ${ }^{9}$. De même, la quasi-absence de politiques en faveur des seniors a des conséquences sur les capacités des seniors à retrouver une situation sur le marché du travail.

\section{Des conditions exigeantes requises par le système de retraite}

En Suisse, le dispositif de protection sociale pour le temps de la retraite a été tardivement mis en place durant la seconde moitié du $X x^{e}$ siècle. Il repose sur trois piliers de protection. Le premier entend garantir le minimum vital par un effort conséquent de solidarité. L'Assurance vieillesse et survivants (AVS) prévoit en effet le versement de rentes minimales et maximales. Ce système par répartition garantit une importante redistribution entre assurés puisque, si les cotisations, proportionnelles au salaire, ne sont pas plafonnées, les rentes le sont dans une proportion de 1 à 2 . Dans les faits, les prestations minimales de l'AVS ne permettent pas d'assurer le minimum vital, au point qu'un important système complémentaire d'aide a été mis en place ${ }^{10}$. Le $2^{\mathrm{e}}$ pilier repose sur des bases également obligatoires, mais sa gestion est de nature privée. Il devrait garantir, dans l'esprit de la loi, le maintien du niveau de vie antérieur. Les cotisations prélevées de façon paritaire sur le salaire sont constitutives d'un capital propre à chaque assuré. Ce système par capitalisation est soumis à surveillance étatique, mais il est géré de façon paritaire au sein de caisses de pension. Une partie de la population ne bénéficie pourtant pas de cette protection en raison de revenus salariaux insuffisants. Les prélèvements ne se font en effet que sur une partie du salaire et varient selon l'âge de l'assuré ${ }^{11}$. Ainsi, les personnes travaillant à temps partiel ou cumulant plusieurs petits emplois ne sont pas assurées. Ce problème concerne surtout les femmes, dont près de une sur deux travaille à temps partiel en Suisse, contre un homme sur sept. Par ailleurs, ce $2^{\mathrm{e}}$ pilier n'est pas obligatoire pour les travailleurs indépendants. Une partie des retraités est contrainte de continuer à travailler après sa retraite officielle, à des conditions souvent non adaptées et peu favorables. Les futurs rentiers sont, en outre, encouragés à constituer un $3^{\mathrm{e}}$ pilier à titre volontaire, pour lequel des avantages fiscaux ont été prévus. Pour les personnes aux revenus les moins élevés, réaliser une telle épargne n'est matériellement pas possible.

En apparence équilibré, le dispositif suisse ne s'est déployé que progressivement, puisque le $2^{\mathrm{e}}$ pilier n'a été rendu obligatoire qu'en 1985 , une partie des générations nées avant 1960 n'en bénéficiant que partiellement. Par ailleurs, il faut relever qu'outre sa complexité, le dispositif helvétique est exigeant en termes de conditions à satisfaire pour en bénéficier pleinement. Ses concepteurs l'avaient bâti sur l'idée que les travailleurs auraient un parcours professionnel linéaire à plein temps, chez un même employeur, sans chômage ou autres risques d'éloignement de l'emploi ; en bref, un parcours typiquement masculin.

9. Dans son rapport (publié en 2017) sur le " chômage des personnes âgées de 50 ans et plus », le Secrétariat d'État à l'économie (Seco) souligne que certains « 60 ans et plus » n'attendent pas d'arriver en fin de droits (aux indemnités journalières de chômage) et optent plutôt pour des retraites anticipées. https://www.seco.admin.ch/dam/seco/fr/dokumente/Arbeit/ALV/Arbeitslosigkeit/Aeltere_Arbeitslose/Bericht_ Arbeitslose_50plus.pdf.download.pdf/FR_Bericht_Arbeitslose_50plus_(2017).pdf

10. Un retraité sur dix est bénéficiaire d'une aidē complémentaire, alors même qu'un tiers des ayants droit n'y a pas recours. Le calcul des prestations complémentaires, variables selon les personnes, résulte d'une estimation effective du minimum vital, en l'absence d'une définition légale de cette notion.

11. Pour le $2^{\mathrm{e}}$ pilier, la loi prévoit un prélèvement sur le " salaire coordonné », compris entre $24675 \mathrm{CHF}$ et 84600 CHF. L'employeur retient sur cette tranche de salaire un montant qu'il doit doubler et verser à l'institution de prévoyance. Le taux de prélèvement croît avec l'âge, par palier. Pour un individu âgé de 25 à 34 ans, le taux du salaire coordonné est de $7 \%$. Ce taux est de $10 \%$ de 35 à 44 ans; de $15 \%$, de 45 à 54 ans; et de $18 \%$, de 55 à 65 ans. 
Ce parcours, relativement idéal, ne correspond pas aux trajectoires professionnelles actuelles, en raison des changements intervenus sur le marché de l'emploi. En effet, une part croissante des hommes est aussi pénalisée en fin de carrière : elle peut pour diverses raisons se retrouver au chômage, être contrainte à l'inactivité professionnelle ou encore à des changements relativement fréquents de places de travail. Par ailleurs, beaucoup de femmes ont un type de carrière rythmé par les événements de la vie familiale. Cette flexibilisation et cette discontinuité de la carrière professionnelle ont pour conséquence de pénaliser les revenus des futurs rentiers.

La vulnérabilité des personnes en fin de carrière, comme des jeunes retraités, est un problème social sous-estimé en Suisse, en dépit du bilan dressé par l'OCDE (2014) à son sujet. Dès lors, il nous paraît utile d'examiner le sens et le poids de certaines de ses recommandations (comme le maintien des travailleurs âgés en emploi par diverses mesures à l'intérieur des entreprises ou l'amélioration de l'employabilité et de la formation continue) et de tenter d'appréhender la nature de l'interface qui existe entre les travailleurs et les entreprises.

\section{Politiques et pratiques des entreprises concernant les « $\mathbf{5 0}$ ans et plus »}

Au-delà du caractère libéral du marché du travail helvétique, l'enjeu réside dans le maintien ou la (ré)intégration des « 50 ans et plus » dans la perspective des politiques et des pratiques des employeurs. La perception qu'ont les seniors de ces dernières les amène aussi à développer des stratégies visant à s'ajuster aux impératifs du marché de l'emploi.

Les politiques et les pratiques des entreprises en relation avec les travailleurs âgés seront abordées suivant deux axes. Le premier met en exergue la question du recrutement des seniors, ce processus faisant apparaître les contraintes auxquelles cette catégorie de travailleurs peut devoir faire face pour être embauchée. Le second axe renvoie en particulier aux pratiques susceptibles de promouvoir le maintien des « 50 ans et plus » en emploi.

Les informations et les données de cette étude ont été recueillies auprès de diverses sources (employeurs, cabinets de recrutement, travailleurs âgés) et au moyen de différentes approches (focus group, enquête Delphi, entretien individuel), l'objectif étant de trianguler les informations émanant de ces différentes provenances. Cette méthode permet de mieux appréhender les difficultés des travailleurs âgés sur le marché du travail. Au-delà des questions de rapports de travail ou de représentations liées à l'âge, la démarche a cherché à tenir compte du rôle des assurances sociales, du chômage et de l'employabilité ou de «l'inemployabilité » des plus de 50 ans.

Les focus groups ont été organisés dans les parties francophone et germanophone de la Suisse. Ils devaient saisir le point de vue des acteurs concernant la situation des seniors sur le marché du travail suisse. Nous avions pour hypothèse que leur position sur le marché s'était détériorée et que certaines conséquences de cette dégradation étaient à attendre, en particulier lors du passage à la retraite. En organisant les discussions au sujet des barrières qui font obstacle à l'intégration ou au retour des travailleurs âgés (50 ans et plus) sur le marché de l'emploi et aux enjeux qui y sont rattachés, ce volet a permis de 
comprendre qui sont les seniors bénéficiaires de mesures du marché du travail $(M M T)^{12}$ ou d'autres dispositifs d'accompagnement selon les cantons. Plus spécifiquement, les parcours empruntés par les seniors ont pu être mis en exergue, tant dans l'environnement professionnel que dans leur vie sociale, dès lors qu'ils ont été contraints de retrouver un emploi. Deux autres aspects sont ressortis des discussions menées dans chaque canton, la question de l'employabilité et celle des difficultés de réinsertion.

La précarité de l'emploi des seniors ainsi que les dispositifs encourageant ou contraignant leur retour à l'emploi ont également fait l'objet de larges discussions entre les acteurs concernés (acteurs publics en charge de l'emploi et de la lutte contre le chômage, employeurs, spécialistes du recrutement, du conseil et du placement, organisateurs de MMT). Les contingences du marché du travail ont ainsi été examinées sous différents angles, ceci afin de percevoir la spécificité des travailleurs âgés en tant que catégorie (en comparaison avec les autres groupes d'âge ou intracatégorielle et par secteur d'activité), de mettre en évidence les ressources et/ou désavantages que présente cette catégorie en termes d'employabilité, ainsi que de montrer les mesures disponibles ou envisagées au profit des seniors. D'autres échanges ont aussi été consacrés aux points de vue des syndicats, des associations de défense des chômeurs, ainsi qu'à l'aspect social de la problématique de la fin de carrière professionnelle.

Confrontés en partie au regard d'experts mobilisés dans le cadre d'une démarche Delphi, ces expériences et témoignages de praticiens permettent de mieux saisir les raisons du maintien ou de la réintégration des « 50 ans et plus » dans la perspective de l'employeur et de mettre au jour sous divers aspects les politiques et les pratiques des entreprises concernant les travailleurs âgés.

\section{En matière de recrutement}

Pour recruter des collaborateurs, les entreprises déploient différentes approches. Certaines s'organisent en interne, avec le concours d'un responsable en charge du recrutement, la décision finale revenant à l'employeur. Les candidatures spontanées sont aussi examinées par ce biais. D'autres entreprises préfèrent confier le processus de recrutement à des instances externes, qu'il s'agisse de cabinets de recrutement, de conseil et de placement ou, selon l'expertise recherchée, de chasseurs de têtes (head hunter) spécialisés dans une branche déterminée. Dans le cas des emplois temporaires, les entreprises - de second œuvre, notamment - recourent aux bureaux de placement et d'intérim, lesquels permettent de trouver du personnel sans que la qualification entre nécessairement en ligne de compte dans la définition du salaire horaire. Le contact avec des individus postulant pour ces emplois temporaires peut également se faire par le bouche-à-oreille (à l'image des communautés portugaises et, plus largement, de celles appelées les «latinos »).

12. Les organisateurs de MMT agissent dans des structures associatives ou privées (souvent des fondations) ayant pour vocation de réaliser des mandats à la demande des services publics. Dans le cas de la promotion de la réinsertion professionnelle, ces acteurs mettent en œuvre les mesures définies avec les ORP. Les chômeurs et les demandeurs d'emploi bénéficient ainsi d'un accompagnement, en termes de formation, de coaching et de conseil, notamment. Les MMT participent à la réalisation du bilan de compétences du chômeur ou du demandeur d'emploi. 
Dans le contexte helvétique, constitué à $80 \%$ de PME, les multinationales ont plutôt tendance à externaliser le processus de recrutement, ce qui comporte à la fois des avantages (rapidité de la procédure ; élargissement de la base de sélection; ouverture à la dimension interculturelle) et des inconvénients (coûts ; important volet administratif en cas de recrutement d'un étranger ; entretiens essentiellement menés à distance, etc.). Du côté des très petites et moyennes entreprises (TPME), l'externalisation est également une tendance forte en raison des besoins de spécialisation. En effet, elles disposent rarement d'un responsable des ressources humaines et la personne qui en fait office se consacre surtout à la gestion du personnel plutôt qu'au recrutement de nouveaux collaborateurs.

Quand une offre d'emploi attire un ou des candidats âgés de plus de 50 ans, le recruteur veille à tenir compte des attentes et exigences du futur employeur influencées par l'âgisme ou les représentations liées à l'âge. Ces représentations, mises en exergue par les comparaisons avec des catégories d'âge inférieures, consistent notamment à déceler chez les collaborateurs et collaboratrices ayant atteint un certain âge (55 ans, voire 50 , parfois même plus jeune) des limites physiologiques, censées restreindre la capacité de travail, induire une faible productivité et entraîner des difficultés d'apprentissage et d'adaptation à certains changements professionnels (Kite, Johnson, 1988 ; Kite, Stockdale et al., 2005 ; Posthuma, Campion, 2007).

Nous avons ainsi relevé une série de filtres qui éloigne les candidats seniors des opportunités d'emploi diffusées sous forme d'annonces. Â côté de ces filtres, existent aussi des limites lors de candidatures spontanées. Cette section concernant le recrutement des seniors est précédée d'une discussion des points de vue et des représentations des professionnels du recrutement, du conseil et du placement à propos des travailleurs âgés. La section suivante se concentre sur le recrutement des seniors. Elle est introduite par une présentation des points de vue et des représentations des professionnels du recrutement, du conseil et du placement des travailleurs âgés.

\section{Les seniors vus par les spécialistes}

Le point de vue des professionnels du recrutement, recueilli lors de nos investigations par l'intermédiaire de focus groups et d'entretiens individualisés complémentaires, montre des attitudes spécifiques susceptibles d'être regroupées en plusieurs catégories ${ }^{13}$.

Entre un militantisme en faveur des seniors et une neutralité de certains recruteurs, c'est le principe de précaution qui prévaut. Ce dernier consiste à situer la barrière du côté du mandant (le potentiel employeur), qui détermine, souvent en amont, une fourchette d'âge dont les recruteurs devront tenir compte pour satisfaire leurs clients. Dans ces circonstances, la marge de manœuvre pour favoriser le dossier d'un senior est très faible. Le choix entre les candidatures en présence est ainsi lié à des critères prédéfinis. Néanmoins, il faut souligner que les compétences et le niveau de salaire restent fortement

13. Les professionnels avaient été invités à répondre, entre autres, aux trois questions suivantes : dans quelles circonstances ou sous quelles conditions procédez-vous au recrutement d'une personne de « 50 ans et plus »? Quels seraient vos critères favorables au recrutement d'une personne de « 50 ans et plus "? Quels seraient vos critères moins favorables au recrutement d'une personne de « 50 ans et plus »? 
déterminants dans la négociation pour un emploi, certains candidats étant prêts à revoir plus ou moins fortement leurs prétentions salariales ou encore à effectuer une formation pour mettre leurs compétences à niveau.

Concernant les arguments favorables au recrutement du senior, si d'aucuns mettent en avant l'expérience, la compétence et la stabilité (voire la loyauté) du senior, d'autres recruteurs prêtent davantage attention à sa flexibilité, sa souplesse face aux changements, de même qu'à sa capacité à s'intégrer dans un système hiérarchisé, notamment quand il faut travailler sous la houlette d'un responsable plus jeune. Les recruteurs considèrent que la flexibilité et la capacité d'intégration dans des équipes où tous les âges se côtoient ne sont pas toujours évidentes et peuvent constituer des freins, notamment en termes d'apprentissage et d'acquisition de nouvelles aptitudes. Mais d'autres obstacles défavorisant le recrutement des seniors ont aussi été évoqués. Il s'agit, par exemple, de l'importance des charges sociales liées au $2^{\mathrm{e}}$ pilier, des compétences en matière de technologies de l'information et de la communication (TIC), d'une possible surestimation de soi («vivre dans le passé »), y compris au niveau des prétentions salariales. Ainsi, si les représentations des recruteurs concernant les travailleurs âgés sont souvent stéréotypées (comme la capacité ou l'incapacité des seniors à s'intégrer dans une nouvelle équipe ou dans un nouvel environnement de travail), ces spécialistes évoquent aussi un grand nombre de contraintes pour le recrutement d'un senior. Elles sont liées aux souhaits des employeurs ou aux règles concernant les cotisations du $2^{\mathrm{e}}$ pilier (coût économique). Ces contraintes et ces représentations déterminent une série de filtres durant le processus de recrutement d'un collaborateur aboutissant au rejet des candidatures de travailleurs âgés.

\section{Les dispositifs pour filtrer les candidatures de seniors}

Dans toute offre d'emploi, un profil et des exigences sont requis en rapport avec les tâches et les responsabilités de la personne recherchée. Ainsi, il n'est pas rare de relever des annonces précisant le nombre de jours de vacances par année conférés au poste à pourvoir. Selon la branche concernée, ce type d'indication peut constituer un facteur bloquant pour la candidature d'un senior, notamment lorsque, dans la convention collective de travail (CCT) concernée, les travailleurs âgés bénéficient d'un nombre de jours de vacances par année supérieur à celui des autres employés. Dans ce cas, le recruteur tiendra compte de l'adéquation ou non entre le nombre de jours de vacances annoncés et le droit aux avantages dont devrait bénéficier un travailleur âgé au titre d'une CCT ${ }^{14}$.

La postulation - ou candidature - requise exclusivement en ligne peut également constituer un filtre en amont, principalement pour les individus ayant des difficultés avec l'outil informatique. Certains postulants font ainsi appel à des aides externes, en commençant par le cercle familial. Les chômeurs et les demandeurs d'emploi inscrits dans un office régional de placement (ORP) peuvent également bénéficier de formation et d'accompagnement de la part des organisateurs de mesures du marché du travail (MMT) ou d'autres structures caritatives. Toutefois, le temps de réaction face à une opportunité d'emploi peut constituer une entrave supplémentaire pour un senior.

14. Selon l'âge du collaborateur, dès 60 ans, les conventions collectives de travail (CCT) vont accorder entre cinq et sept semaines de vacances selon les branches économiques. 
L'importance croissante des candidatures en ligne souligne la pertinence de l'observation d'un spécialiste d'une agence internationale de recrutement, de conseil et de placement ${ }^{15}$. Selon lui, en effet, la propension au recrutement en ligne est telle que, vu le nombre d'actifs disposant tout au plus d'un certificat fédéral de capacité (CFC) ${ }^{16}$, les systèmes informatiques sur lesquels s'adossent les grands groupes tendent à écarter les candidatures des « 50 ans et plus ». De fait, ces dispositifs opèrent un premier filtrage, par exemple, en ne prenant pas en considération les niveaux de qualification inférieurs au bachelor, parce que le CFC est inconnu et non enregistré dans ces systèmes. Les générations actuelles de travailleurs âgés ont en moyenne une formation moins élevée, comparées aux générations d'actifs plus jeunes. De nombreux seniors sont entrés sur le marché du travail dans les années 1960 ou 1970 à la suite d'une formation professionnelle par apprentissage, très développée en Suisse. Une partie de cette population ne dispose pas de formation spécifique ou initiale et n'a pas acquis depuis d'autres formations diplômantes. Les uns et les autres se retrouvent ainsi bloqués par de tels systèmes ou des procédures pour lesquelles le niveau de qualification constitue un critère d'embauche primordial.

Un exemple éloquent illustre le cas de figure d'une formation qui ne correspondrait pas ou plus à celle exigée par l'employeur. La suppression du secret bancaire pour les portefeuilles non suisses a en effet provoqué la perte d'emploi d'un grand nombre de gestionnaires de fortunes, en raison de la réduction du volume de leurs activités liée au départ de la Suisse de certains clients qui cherchent à placer leur argent dans des paradis fiscaux. Malgré une longue carrière professionnelle, ces personnes titulaires d'un CFC unique ont été obligées d'envisager une réorientation professionnelle, alors qu'elles étaient seulement à quelques années de l'âge de la retraite ordinaire.

Durant la procédure de recrutement, apparait souvent un autre obstacle : la difficulté d'obtenir un entretien avec un responsable de l'entreprise choisie suite à une candidature spontanée ou à la publication d'une offre d'emploi. Obtenir un contact interne par ses propres démarches est peu évident pour un demandeur d'emploi. Du côté de l'employeur, les entretiens qui découlent d'un processus de recrutement sont réalisés à un moment opportun pour l'entreprise. Au niveau des offices régionaux de placement (ORP), de même que pour certains organisateurs de MMT, les seniors demandeurs d'emploi doivent également tenir compte du comportement et de l'image qu'ils renvoient à leurs interlocuteurs. Selon ces acteurs, l'attitude du candidat revêt une importance cruciale pour convaincre et susciter un entretien pouvant déboucher sur une embauche. Elle renvoie notamment à la motivation, à la posture et à la prestance individuelles en matière de recherche d'emploi ${ }^{17}$. Par rapport à l'effet du salaire comme exigence, Patrick Arni (2012) parle d'ailleurs de salaire minimum « encore juste » que le demandeur d'emploi

\footnotetext{
15. Intervention effectuée lors du focus group $\left(\mathrm{n}^{\circ} 2\right)$ consacré aux « barrières à l'intégration ou au retour des travailleurs âgés (50 ans et plus) sur le marché du travail et les enjeux liés à cette question », Unil, le 28 mai 2015.

16. Le certificat fédéral de capacité (CFC) sanctionne une formation professionnelle initiale par un apprentissage dont la durée est de trois ou de quatre ans. II jouit d'une très bonne reconnaissance au sein de la population et auprès des employeurs.

17. En 2007 déjà, le rapport Chômage de longue durée : situation et mesures de l'Observatoire du marché du travail Suisse orientale, Argovie et Zoug (Amosa) engageait les offices régionaux de placement (ORP) et les professionnels impliqués dans la lutte contre le chômage de longue durée à tenir compte de ces « facteurs mous » dans la dynamique d'encadrement et d'appui aux demandeurs d'emploi. www.amosa.net/fileadmin/user_upload/projekte/LZA/02_LZA_Schlussbericht_FR.pdf
} 
pourrait définir comme « limite inférieure subjective » au-dessous de laquelle l'emploi n'est plus considéré comme financièrement intéressant.

Face à une croissance - lente mais régulière - du nombre de seniors au chômage, surtout de longue durée, signe de leurs difficultés à se réinsérer, des instruments politiques sont mis en place par les cantons pour encourager leur intégration. Les demandeurs d'emploi seniors tout comme les employeurs peuvent ainsi y recourir. C'est le cas notamment des allocations d'initiation au travail (AIT) ${ }^{18}$ ou des allocations de retour à l'emploi (ARE) ${ }^{19}$ qui développent un volet spécifique pour les « 50 ans et plus ».

Cependant, certains acteurs restent dubitatifs en considérant qu'à compétences égales entre une personne âgée de 45 ans et une autre de 62 ans, « le senior n'a de chance que si le plus jeune ne $s^{\prime} y$ connaît pas trop par rapport au poste ${ }^{20} \gg$. Les défenseurs de ce point de vue mettent principalement en avant le coût du salarié âgé, sa capacité de travail (aspects physiologiques et obsolescence des compétences) et la courte durée d'activité qui lui resterait avant de partir à la retraite. La pertinence de telles observations peut être questionnée, surtout quand la tendance actuelle dans les trajectoires professionnelles est caractérisée par une très grande mobilité ainsi que par des parcours non linéaires. Avec une durée de 5 à 10 ans chez un même employeur, la mobilité du jeune travailleur devient presque similaire au temps de parcours qu'il reste à un travailleur âgé pour atteindre l'âge de la retraite ordinaire ${ }^{21}$. L'évaluation des investissements à consentir pour l'adaptation d'un travailleur jeune ou d'un collaborateur âgé n'est pas forcément au désavantage du second, si le contexte est exempt d'âgisme.

C'est là une piste pour saisir la propension, chez certains employeurs, à privilégier les ressources particulières du senior qui se conjuguent en termes de fiabilité, d'expérience et de vecteur de pérennité (capacité de transmettre des savoirs et du savoir-faire).

\section{Employeurs et candidatures spontanées des « 50 ans et plus »}

Dans leurs démarches de recherche d'emploi - volontaires ou par activation d'un réseau (Dang, Zajdela, 2009 ; Barbier, 2002) -, certains seniors contactent directement l'employeur qu'ils ont ciblé en fonction de la branche et par l'intermédiaire duquel ils espèrent trouver un poste.

18. L'AIT est une prestation de l'assurance-chômage ayant pour objet le financement d'une partie du salaire durant la phase d'initiation au travail d'un nouveau collaborateur recruté en CDI (exceptionnellement, en CDD de 12 mois minimum), laquelle peut durer jusqu'à 6 mois. Pour les « 50 ans et plus », le versement (dégressif) de la compensation financière peut aller jusqu'à 12 mois. L'AIT est couplée à la formation continue. Initiée par le canton de Vaud, l'AIT s'étend progressivement aux autres cantons (Genève, Neuchâtel, Valais, etc.).

19. Par cette mesure, l'employeur qui recrute en CDI un chômeur en fin de droits, un ex-indépendant ou une personne bénéficiaire de l'aide sociale, reçoit de l'État de Genève une participation (dégressive) moyenne de $50 \%$ du salaire brut. Pour les personnes de « 50 ans ou plus ", ce financement d'une partie du salaire peut s'étaler à 24 mois consécutifs.

20. Propos de monsieur D., directeur d'un cabinet de conseil en management sis en Valais, lors du focus group $\left(\mathrm{n}^{\circ} 2\right)$ consacré aux « barrières à l'intégration ou au retour des travailleurs âgés ( 50 ans et plus) sur le marché du travail et les enjeux liés à cette question », université de Lausanne, le 28 mai 2015.

21. D'après l'OFS, entre 2010 et 2016 , les différents groupes d'âge présentent une mobilité professionnelle (hors changements d'emploi à l'intérieur d'une même entreprise) suivant les moyennes ci-après : $20 \%$ pour les $15-24$ ans, $11,1 \%$ chez les $25-39$ ans, $5,7 \%$ chez les $40-54$ ans et $2,5 \%$ chez les $55-64$ ans. Voir «Taux de rotation net selon les caractéristiques des personnes actives occupées et des entreprises, en pour cent, ESPA, 1993-2017 ». www.bfs.admin.ch/bfs/fr/home/statistiques/travail-remuneration/ activite-professionnelle-temps-travail/actifs-occupes/mobilite-professionnelle.html 
Face à ces candidatures spontanées, les employeurs réagissent différemment selon la situation de l'entreprise et le profil du candidat. De nos entretiens, il ressort ainsi qu'une grande majorité des candidatures spontanées de seniors demandeurs d'emploi ne reçoit aucune réponse (ni confirmation de réception de courrier ni aucune autre forme de réponse ou d'information). Pour avoir des informations concernant leurs candidatures, les seniors doivent appeler et relancer eux-mêmes les services concernés. Ceux qui reçoivent un courrier en retour ont rarement un avis favorable. Non pas que les profils soient inintéressants, mais parce que la candidature, pour être retenue, doit être réceptionnée au « bon » moment, c'est-à-dire lorsque l'entreprise prévoit un poste à pourvoir. Dans ces circonstances, bénéficier d'un réseau peut jouer un rôle déterminant. Cela permet de disposer d'indices sur le moment opportun pour déposer sa candidature, et bénéficier d'informations « de l'intérieur » peut avoir un effet décisif sur le succès d'une démarche de candidature ${ }^{22}$. L'étude de Bonoli et al. (2013) permet tout de même de nuancer cette assertion. Elle révèle que le réseau social est peu déterminant pour retrouver un emploi dans le cas des chômeurs qualifiés, tandis qu'il peut être très utile pour les chômeurs peu qualifiés ou dans des secteurs ne nécessitant pas forcément une grande qualification (agriculture, construction, hôtellerie et restauration).

La candidature spontanée d'une personne de 50 ans ou plus paraît ainsi peu pertinente pour entrer en contact avec un employeur. Elle reste toutefois une voie prônée et encouragée par les ORP et leurs conseillers en placement. Pour des baby-boomers qui n'avaient pas éprouvé le besoin de constituer jusqu'alors un dossier de candidature pour un emploi, l'envoi d'une candidature spontanée suppose d'avoir « fait le deuil ${ }^{23}$ » sinon de l'activité précédente du moins de l'environnement et des conditions professionnelles inhérentes à cet emploi. Faire ce deuil s'entend ici en termes d'attitudes et de motivation à retrouver du travail. À ce stade, les hommes et les femmes manifestent des attitudes distinctes, même si celles-ci ne sont pas propres au genre, certaines étant observées autant chez les hommes que chez les femmes. Les témoignages que nous avons recueillis auprès des « 50 ans et plus » montrent que les femmes - surtout celles qui sont célibataires ou divorcées, vivant avec ou sans enfant mineur - ont tendance à se replier sur elles-mêmes. Cet isolement peut aboutir à les écarter de tout espace pouvant favoriser du lien social. D'un côté, ces personnes peuvent chercher à préserver une certaine image de soi liée aux représentations intériorisées du travail comme valeur de référence dans une société fortement individualisée. Ces représentations se conjuguent difficilement avec le chômage, surtout lorsqu'il perdure. De l'autre, la personne perd progressivement de son pouvoir d'achat lorsque le chômage perdure. À l'exemple de cette Romande, juriste, célibataire et mère d'un enfant mineur, qui renonce à s'offrir une bière dans un espace de rencontre (un café-restaurant, par exemple), sa situation financière l'obligeant à une gestion parcimonieuse de ses indemnités de chômage. De même, cette architecte designer qui, quelques années après son divorce, ne va plus en vacances avec ses filles, faute de moyens pour satisfaire certains besoins essentiels du ménage.

Chez les hommes, le repli sur soi est plutôt une posture adoptée lorsqu'ils sont mariés, plus particulièrement, $s^{\prime}$ ils vivent une crise familiale marquante (comme une procédure de

22. La question du type de réseau et de son rôle a fait l'objet de nombreuses investigations à partir des travaux de Mark Granovetter (Granovetter, 1974). L'importance cruciale des réseaux a été récemment mise en évidence par une large enquête menée dans le canton de Vaud (Turtschi, 2015).

23. Extrait du focus group $n^{\circ} 1$ consacré aux « organisateurs de mesures actives du marché du travail et d'autres structures d'appui à l'insertion professionnelle », Unil, 26 et 27 mai 2015. 
divorce ou des brouilles avec les enfants). Ces tensions les amènent à être inquiets autant pour leur statut social que pour leur rôle de père, l'un comme l'autre étant susceptible d'être remis en cause lorsque la durée du chômage s'allonge. Le retour à l'activité constitue un impératif de reconquête du statut social fragilisé et un facteur d'apaisement du climat social, notamment dans l'espace familial. Toutefois, la réintégration, tout comme le maintien en emploi des 50 ans et plus, nécessite des dispositions moins suspicieuses des employeurs à l'égard des seniors. Cela implique aussi une posture plus active de leur part, à travers de vraies pratiques de gestion des collaborateurs âgés.

\section{En matière de promotion du vieillissement actif}

\section{Des bonnes pratiques malgré les limites du système de prévoyance}

Après la votation du 9 février 2014 contre l'immigration de masse ${ }^{24}$, les dirigeants de la multinationale pharmaceutique bâloise Novartis se sont davantage intéressés aux travailleurs âgés afin de constituer une alternative aux travailleurs immigrés qualifiés qui étaient recrutés jusqu'alors. Le directeur des ressources humaines s'est ainsi positionné en faveur de solutions flexibles pour les seniors, tout en soulignant que «le système de prévoyance ne facilite pas l'emploi des seniors ${ }^{25}$ » en raison des charges sociales élevées.

En plus d'un projet nommé Spark Arena, qui consiste à mettre en place un espace de rencontre entre personnes d'âges différents et de départements distincts, Novartis a également développé l'initiative Prime force. Lancée en 2007, elle vise essentiellement à créer les conditions d'intéressement des seniors cadres en leur laissant le choix de prolonger leur activité ou pas à l'approche de la retraite, sur la base d'un consentement mutuel comme principe fondamental. Ce faisant, ceux qui optent pour suivre cette mesure Prime force doivent donner leurs préférences en termes de temps et de type de travail et préciser ce qu'ils en attendent. En retour, ce dispositif offre des accords-cadres avec des conditions souples. Par exemple, sur le plan financier, les seniors cadres restant dans la même activité bénéficient d'une rémunération constante, celle-ci étant convertie en jours et heures plutôt qu'en termes de salaires mensuels ${ }^{26}$. S'ils changent d'activité ou de poste à l'intérieur du groupe, une autre rémunération est définie.

En janvier 2015, la moitié des seniors cadres engagés dans le contexte de Prime force touchait $50 \%$ de leurs pensions de retraite, puisqu'ils n'étaient que partiellement retraités ; $30 \%$ percevaient $100 \%$ de leurs pensions de retraite et $20 \%$ touchaient entre $10 \%$ et $25 \%$ de la pension de retraite. L'employeur s'attend à une forte expansion du nombre d'employés inscrits dans ce dispositif, plus particulièrement si ces derniers peuvent bénéficier de services supplémentaires avant la retraite. Ceux-ci se déclinent sous forme

24. Votée par le peuple suisse le 9 février 2014, la loi contre l'immigration de masse mettait l'accent sur trois lignes forces : la mise en place d'une loi prévoyant des quotas de permis de travail pour les frontaliers ainsi que des quotas pour les demandeurs d'asile ; la remise en place de quotas pour les travailleurs étrangers au bénéfice d'un permis de résidence (permis $L, B$ et $C$ ) ; la réintroduction de la préférence nationale consistant à obliger les entreprises suisses à recruter prioritairement des travailleurs de nationalité suisse.

25. Extrait de l'intervention du Dr Thomas Bösch, directeur des ressources humaines suisses (Novartis), à l'occasion de la conférence de presse sur l'initiative «Avenir du marché suisse du travail » organisée à Berne le 21 janvier 2015. Cette initiative est une œuvre commune de l'Union patronale suisse et d'Économie suisse.

26. Ne correspondant plus à un salaire, la rémunération perçue équivaut à l'indexation fixée pour un même poste de travail mais calculée et versée suivant les heures effectivement ouvrées. 
de conseils en matière de préparation à la retraite de la part de la firme, de possibilités de travail par projet, ou encore de retraite flexible. Pour les seniors cadres inscrits dans cette mesure, la motivation financière est plutôt secondaire : rester en forme, être en bonne santé, avoir une activité constructive et motivante constituent des composants plus importants à leurs yeux.

Tout comme Novartis, d'autres grandes entreprises développent ce type d'initiative. Dans le parapublic, les Chemins de fer fédéraux (CFF) ont mis en place le modèle Activa. Une des particularités de cette initiative réside dans le fait que les CFF offrent une durée de trois années au maximum pour les seniors désireux de prolonger leur carrière professionnelle.

Mis à part quelques cas très spécifiques, ces opportunités ne se déclinent pas de la même manière chez les femmes et chez les hommes. Même si celles-ci restent de plus en plus tardivement en activité (élévation progressive de l'âge de la retraite ; maintien de l'activité professionnelle pendant et après l'éducation des enfants), l'Office fédéral de la statistique (OFS) notait aussi qu'elles avaient un taux d'activité professionnelle « sensiblement plus bas que celui des hommes [âgés] entre (...) 30 et 45 ans et à partir de [l'âge de] 55 ans $^{27}$ ». Par ailleurs, lorsque le retour à l'emploi des femmes s'effectue par le biais d'une activité à temps partiel ou par des emplois précaires, les initiatives comme celles des grands groupes leur deviennent presque inaccessibles. De plus, l'OFS (op. cit., p. 14) n'a pas manqué de relever que les femmes sont plus souvent que les hommes dans les positions de salariés sans fonction dirigeante « même lorsque hommes et femmes ont un niveau de formation égal ${ }^{28} \gg$. Ainsi, ces opportunités restent davantage accessibles aux hommes.

Ces formes d'incitation ne sont pas les seules à être appliquées par les employeurs. La bonification par l'âge constitue une autre pratique, qui fait cependant l'objet de profondes réflexions de la part des partenaires sociaux ainsi que des acteurs politiques.

\section{Les traitements salariaux et la bonification par l'âge}

Le maintien des seniors en emploi dépend en partie des conditions générales qui prévalent en fin de carrière. Sans les nommer explicitement, les recommandations de l'OCDE demeurant générales, les charges sociales croissant avec l'âge des travailleurs constituent un obstacle à la réinsertion, et cela dès 55 ans. Plusieurs interventions parlementaires ont tenté de remédier à cette situation qui est liée à l'entrée en vigueur progressive de la loi, à partir de 1985.

Après le postulat Polla (02.3208), en 2002, et celui du Parti démocrate chrétien (PDC) (05.3651), en 2006, le Parlement a demandé au Conseil fédéral de présenter un rapport sur les « différentes possibilités et toutes autres mesures incitatives » qui permettent

27. OFS, «Vers l'égalité entre femmes et hommes. Situation et évolution », Situation économique et sociale de la population (617-1300), Neuchâtel, 2013. Disponible sur le site de l'Office fédéral de la statistique : https://www.bfs.admin.ch/bfs/fr/home/statistiques/situation-economique-sociale-population/ egalite-femmes-hommes/activite-professionnelle/situation-profession.html

28. Dans son rapport Femmes d'affaires et femmes cadres. Une montée en puissance, publié en janvier 2015, le BIT montrait aussi qu'en Suisse, le pourcentage de femmes cadres était passé de moins de $25 \%$ en 2000 à 31,4 en 2012. Et, avec 33,2 \% de femmes cadres moyens et cadres supérieurs - tous postes de direction confondus -, en 2012, la Suisse se positionnait au $44^{\mathrm{e}}$ rang mondial sur 126 États concernés par l'étude. 
de favoriser l'accès ou le maintien en emploi des personnes de plus de 55 ans. Il s'agit notamment : « d'harmoniser les pourcentages du salaire coordonné correspondant aux bonifications de vieillesse entre les différentes classes d'âge, de façon que le taux appliqué à la classe d'âge au-dessus de 55 ans soit inférieur à ce qu'il est actuellement [en 2006]. La possibilité que le taux soit le même pour toutes les classes d'âge, en tout cas pour la part de l'employeur, devrait également être évaluée ».

Des observations faites par le Conseil fédéral, il ressort néanmoins qu'un réajustement de l'échelonnement n'améliorerait pas particulièrement la situation des seniors sur le marché de l'emploi. Des mesures plus favorables ont ainsi été préconisées, notamment au travers d'un assouplissement des conditions-cadres dans les $1^{\mathrm{er}}$ et $2^{\mathrm{e}}$ piliers. Une réforme dite « Prévoyance professionnelle 2020 » visait à introduire un certain nombre de modifications dans ce sens. Elle a été rejetée en votation populaire en 2017.

Contrairement aux organisations syndicales qui n'excluent pas les possibilités de lissage des bonifications de vieillesse, les instances patronales s'adossent sur la posture du Conseil fédéral pour s'opposer à toute directive visant à obliger les employeurs à développer des mesures de protection des travailleurs âgés. En effet, du point de vue des employeurs, la mise en place de mesures ou de directives en faveur des « 50 ans et plus » est jugée contre-productive, un chef d'entreprise ayant toujours la possibilité de se séparer de son collaborateur peu avant cet âge. Cette posture coïncide avec celle de Bernhard Weber, chef suppléant du secteur Analyse du marché du travail et politique sociale au Secrétariat d'État à l'économie (Seco). En effet, dans un article publié par le magazine La Vie économique ${ }^{29}$, il constate que « les actifs âgés [sont] moins touchés par des ruptures involontaires des contrats de travail que les jeunes » et, dès lors, « il n'apparaît pas très opportun de vouloir améliorer la situation de ce groupe en le protégeant par des dispositions spécifiques contre les licenciements liés à l'âge. Cela pourrait même constituer un obstacle supplémentaire à l'engagement de travailleurs âgés ». Cet obstacle pourrait se présenter notamment en termes de licenciement préventif, de non-renouvellement systématique du contrat des plus de 50 ans, ou encore en termes d'offres d'emploi formatées en emplois atypiques. La notion d'encouragement est ainsi préférée aux directives ou autres mesures discriminatoires alors que, dans le débat politique, ces dernières seraient en partie considérées comme favorables à l'intégration des seniors.

Des initiatives pour les seniors ayant pour vocation d'inciter à l'embauche existent cependant. Ces encouragements consistent d'abord à faire assumer par les pouvoirs publics tout ou partie des charges sociales des bénéficiaires engagés. Cependant, ces mesures ont été peu utilisées en Suisse, compte tenu de la complexité des processus de décision et du contexte peu favorable à ce type d'intervention. Quelques contreexemples existent pourtant, comme dans le canton de Neuchâtel, où le Service de l'emploi a mis en place une initiative permettant la prise en charge de la part patronale au $2^{\mathrm{e}}$ pilier $^{30}$.

29. $N^{\circ} 7,2015$. https://dievolkswirtschaft.ch/content/uploads/2015/06/22 Dossier FR.pdf

30. "Cette mesure incitative est limitée à 520 francs suisses par mois, au maximūm, pour une période de 12 à 24 mois selon l'âge (12 mois pour les demandeurs d'emploi de 50 à 54 ans, 18 mois pour les demandeurs d'emploi de 55 à 59 ans et 24 mois pour les demandeurs d'emploi de 60 ans et plus). ” 


\section{La formation continue}

Les travailleurs âgés - surtout ceux qui ont connu le chômage durant la dernière partie de leur carrière - rencontrent d'importantes difficultés à se maintenir ou à réintégrer le marché du travail. En cause, selon les employeurs, l'obsolescence des compétences dans un contexte d'évolution technologique de plus en plus marquée. De telles constatations ont également conduit l'OCDE à insister sur l'enjeu de la formation continue en fin de carrière professionnelle, notamment sous le prisme d'une dynamique préventive favorisant le vieillissement actif tout au long du parcours professionnel (Guillemard, 2013).

Ainsi, parmi les pistes de réflexions de l'OCDE au sujet de la Suisse, on trouve celles visant l'amélioration de l'employabilité des seniors pour leur offrir de meilleures chances sur le marché du travail. Une des réponses privilégiées est celle de la formation continue qui nécessite une prise de conscience importante au sein de l'ensemble de la population suisse. En effet, pour les personnes à qualification supérieure, la Suisse présente le meilleur bilan de l'ensemble des pays considérés. À l'inverse, pour les personnes ayant un faible niveau de formation, l'incitation à suivre une formation continue est faible et, dans tous les cas, nettement inférieure aux autres groupes (OCDE, 2014).

Cette situation paradoxale montre l'importance de sensibiliser l'ensemble des travailleurs à leur adéquation au marché de l'emploi. Les principaux obstacles résident, d'une part, dans l'absence ou l'inadéquation de la formation de base, en particulier chez une partie des travailleurs immigrés et, d'autre part, dans l'hétérogénéité extrême des situations rencontrées, chez les femmes notamment. Enfin, le financement de la formation continue représente un investissement dans le personnel dont l'employeur espère pouvoir tirer profit. Ce type de calcul entraîne une profonde divergence de points de vue entre les partenaires sociaux. Ainsi, pour l'Union patronale suisse, les travailleurs âgés ont une importance primordiale compte tenu de la pénurie de main-d'œuvre qualifiée et du frein mis à l'immigration par la votation du 9 février 2014. Mais, pour les employeurs, il incombe d'abord aux travailleurs de développer « leurs qualifications techniques et personnelles, afin de maintenir leur employabilité. En contrepartie, les employeurs et les pouvoirs publics soutiennent la formation et le perfectionnement des travailleurs âgés dans la mesure où l'exploitation de l'entreprise le permet ${ }^{31}$ ».

Nos recherches sur « la fin de carrière professionnelle » nous ont permis d'observer l'hétérogénéité des formations initiales, regroupées en quatre grands types de situations de formation chez les travailleurs âgés. Certains seniors n'ont jamais effectué de formation certifiée ou diplômante, d'autres n'ont que le certificat fédéral de capacité (CFC) comme preuve d'une formation certifiée. Il y a, par ailleurs, des seniors détenteurs d'au moins un diplôme universitaire ou équivalent, alors que d'autres sont détenteurs d'un diplôme post-grade, voire d'un doctorat (tableau 1).

Dans notre échantillon, l'absence de formation particulière est plus manifeste chez les hommes. Les femmes sans qualification sont celles qui ont interrompu très tôt leurs cursus de formation et ont intégré le marché du travail de façon précoce. Par la suite, elles n'ont pas valorisé leurs compétences ou leur savoir-faire par l'acquisition d'une qualification

31. Valentin Vogt, président de l'UPS, cité in La Vie économique, op. cit., p. 60. 


\begin{tabular}{|c|c|c|c|}
\hline Niveau de formation & Femmes & Hommes & Total \\
\hline Sans formation & 1 & 2 & 3 \\
\hline Sans CFC & 7 & 2 & 9 \\
\hline CFC ou baccalauréat & 6 & 12 & 18 \\
\hline CFC + autres certificats/diplômes & 6 & 15 & 21 \\
\hline Études supérieures hors PhD (doctorat) & 6 & 14 & 20 \\
\hline $\mathrm{PhD}$ (doctorat) & 2 & 3 & 5 \\
\hline
\end{tabular}

Source : recherche Fonds national suisse de la recherche scientifique (FNS), « Fin de carrière professionnelle : enjeux suisses d'une situation en voie d'ébullition ", 2016.

spécifique ${ }^{32}$. Les « 50 ans et plus » ont bénéficié du contexte économique de pleinemploi des années d'après-guerre. Un grand nombre d'actifs a donc trouvé du travail sans difficulté malgré des lacunes en termes de capital culturel et de qualifications pour satisfaire aux exigences du marché du travail actuel.

Ces observations doivent être replacées dans un contexte plus large. En 2008, l'OFS soulignait déjà que, malgré des améliorations indéniables, le niveau de formation des 50-64 ans restait encore faible comparé à celui des 30-49 ans $\mathrm{s}^{33}$. En revanche, de manière plus récente, le Seco notait en 2016, qu'entre autres « caractéristiques des chômeurs enregistrés selon leur classe d'âge », la comparaison entre les « 50 ans et plus » et les 25-49 ans indiquait que 1,3\% des seniors n'avaient pas achevé l'école obligatoire et que $48,5 \%$ avaient atteint le degré secondaire, contre respectivement $0,8 \%$ et $46,1 \%$ des 25-49 ans. Si les différences de niveau de formation se sont estompées durant le début des années 2010 , seuls $21,4 \%$ des seniors au chômage avaient obtenu une qualification de niveau tertiaire, contre $69 \%$ des $25-49$ ans.

Un autre phénomène touche aujourd'hui un grand nombre de baby-boomers qui ont connu la sécurité d'emploi par une longue expérience professionnelle effectuée chez le même employeur. Cette fidélité à l'entreprise - signe contextuel de stabilité et de loyauté - se révèle subitement désavantageuse dès lors que la relation professionnelle prend fin. La recherche d'un emploi est donc pour ces personnes un apprentissage totalement nouveau.

Quels que soient le niveau de formation atteint et la trajectoire professionnelle suivie, la formation continue peut contribuer à améliorer l'employabilité des actifs âgés, en les préparant à de nouveaux métiers, par exemple. Toutefois, cette possibilité soulève un double questionnement : à quel moment doit-elle être entamée et se poursuivre ? Qui doit la financer et/ou en assumer les incidences ou les effets?

32. Pour les personnes soucieuses de la valorisation des acquis de l'expérience (VAE) au titre de la formation professionnelle, le financement de la procédure varie entre 4500 et 6000 euros, sachant aussi qu'en cas de succès, l'expérience peut n'être que partiellement reconnue.

33. Office fédéral de la statistique (OFS), «L'activité professionnelle des personnes de 50 ans et plus », Actualités OFS, Neuchâtel, février 2008. 
Lors de la seconde conférence nationale sur les travailleurs âgés, en avril 2016, « l'acquisition d'une formation professionnelle continue et de qualifications supérieures par les adultes » a été considérée comme un impératif ${ }^{4}$. Un consensus semble s'être dégagé du côté des partenaires sociaux. Néanmoins, si les CCT peuvent servir de cadre pour favoriser la formation continue de tous les travailleurs, pour les actifs âgés désireux de réintégrer le marché de l'emploi, la problématique se pose d'une façon autrement plus complexe. Faut-il mettre à jour ses compétences dans un même domaine, sans la garantie de retrouver un emploi dans la même branche ? Faut-il, au contraire, se diversifier en vue d'élargir ses compétences, au risque de buter sur un employeur dubitatif quant à la durée d'intégration et d'adaptation ? Ou encore faut-il plutôt promouvoir de nouvelles compétences en termes de réorientation professionnelle?

Dans la plupart des cantons, il existe des mesures pour favoriser la formation et la réintégration des demandeurs d'emploi âgés. Certaines passent par des organisateurs de MMT. Or, il y a parfois un problème de correspondance entre la formation ciblée par le demandeur d'emploi - au regard de ses aspirations - et l'offre de formation que veut bien financer le service public. Dans l'autre sens, les mesures définies en rapport avec les MMT ne coïncident pas toujours avec les réels besoins en formation des actifs âgés. Il faut d'abord trouver un emploi, certes, mais élaborer un CV ou se motiver à nouveau ne constitue qu'une séquence du processus pour améliorer l'employabilité de l'actif âgé. Les seniors que nous avons interrogés nous précisent d'ailleurs que les MMT pourraient davantage investir dans les besoins réels de formation des personnes qu'elles accompagnent, sachant aussi que cela représente un coût pour les finances publiques.

En outre, un dispositif comme l'allocation d'initiation au travail (AIT) s'avère peu attrayant pour les employeurs. En effet, malgré les possibilités offertes, le recrutement de seniors par ce dispositif de l'assurance-chômage est encore assez faible. Il faut alors se demander si ce n'est pas le type de contrat qui est dissuasif. En effet, conditionner l'AIT à un CDI (même s'il y a possibilité de recruter sur un CDD pour certains cas) peut très vite décourager un employeur qui ne voudrait pas se risquer à assumer les lacunes potentielles du demandeur d'emploi ${ }^{35}$.

La question du financement de la formation continue demeure encore un enjeu de taille. La contribution (financière, aménagement des conditions de travail) par le travailleur lui-même s'avérerait nécessaire pour inciter l'employeur à l'inscrire dans la gestion prévisionnelle des emplois et des compétences (GPEC) ${ }^{36}$. Et si cette question du financement est un problème en soi, elle ne doit pas en occulter d'autres. Des mesures de soutien et d'encouragement restent tout autant nécessaires pour une intégration de la formation dans le parcours professionnel du travailleur. À ce titre, en raison de l'indisponibilité potentielle au poste de travail durant le temps de formation, le dialogue avec l'employeur doit permettre de déterminer les conditions pratiques de la mise en œuvre de la formation continue. Il doit dès lors également mobiliser l'ensemble des personnes concernées, en particulier celles qui sont en fin de carrière, qu'elles travaillent à temps partiel ou qu'elles soient en emploi précaire.

34. Selon le conseiller fédéral, Johann N. Schneider-Ammann, ministre de l'Économie (La Vie économique, $n^{\circ} 7$, 2015, pages 57-58). http://dievolkswirtschaft.ch/content/uploads/2015/06/DV 72015 Gesamt Web FR.pdf 35. En Suisse, la période d'essai peut varier d'un à trois mois et elle s'obšerve seūlement pour les CDI. 36. En Suisse, on estime qu'environ la moitié des dépenses de formation continue est assumée par les demandeurs eux-mêmes et les femmes paieraient plus que les hommes (La Vie économique, $n^{\circ} 6,2009$ ). 
Par ailleurs, si la formation doit être appréhendée comme un atout pour l'entreprise, en permettant au personnel de s'adapter aux changements dans les modes de production, elle doit également constituer un avantage pour l'employé. En améliorant son profil et ses capacités à travers l'acquisition de nouvelles compétences, le développement personnel, les opportunités de carrière et de mobilité, ou encore un bilan de compétences, il est alors davantage en mesure de changer de poste, que cela soit contraint ou souhaité.

La formation continue constitue donc un véritable enjeu de productivité au travail. Un investissement est attendu de la part de l'employé et de l'employeur, mais c'est surtout dans la durée que s'inscrit sa pertinence. Dès lors, le maintien en activité et la limitation du turn-over deviennent un enjeu pour l'employeur qui doit évaluer lequel du jeune candidat ou du senior sera recruté et/ou promu. Une telle perspective devrait particulièrement favoriser l'insertion des femmes, notamment après une période d'arrêt non liée à une démission ou à une autre forme d'arrêt volontaire.

\section{Le licenciement}

Le cadre du marché libéral n'incite pas les organisations patronales suisses à envisager des mesures tendant à protéger les travailleurs contre les licenciements. Même si le dialogue social est très fortement cultivé chez les différents partenaires sociaux, les employeurs veillent à conserver une marge de manœuvre afin que le critère économique puisse demeurer l'élément central de viabilité de leur entreprise. Dès lors, une protection des collaborateurs contre les licenciements leur paraît contre-productive et pourrait les inciter à ne plus embaucher de seniors, voire à les licencier de manière préventive. C'est à ce titre que, malgré leur sensibilité à la problématique des travailleurs âgés, les différentes faitières ${ }^{37}$ des employeurs rejettent toute velléité de mesures allant dans le sens d'une protection des seniors contre le licenciement ${ }^{38}$.

Au demeurant, cette posture patronale n'est pas spécifique à la catégorie des « 50 ans et plus ». Dans le droit du travail suisse, le code des obligations (art. $336 \mathrm{CO}$ ) interdit tout licenciement jugé abusif, ainsi nommé s'il était prononcé pour une raison inhérente à la personnalité de l'autre partie, comme l'âge, la religion, la race, l'orientation sexuelle (art. $336 \mathrm{CO}, 1^{\mathrm{er}}$ al., lettre a). Sur ce fondement, aucun employeur n'admettrait avoir ciblé, licencié ou refusé de renouveler le contrat d'un collaborateur pour des motifs liés à l'âge ${ }^{39}$. Formellement, ce sont d'autres raisons qui seraient invoquées, en lien avec la conjoncture économique, les changements d'orientation dans la production, les crises, etc.

37. En raison du fédéralisme, les associations professionnelles suisses se sont constituées en organisations faîtières afin d'élaborer des positions communes dans les débats politiques et sociaux.

38. Peu avant la première conférence nationale (avril 2015) consacrée aux travailleurs âgés, Daniella Lützelschwab, membre de la direction de l'Union patronale suisse, publiait un article dans l'organe de l'association. Elle y notait que « l'Union patronale suisse s'oppose aussi bien à un renforcement de la réglementation de la protection contre les licenciements et à l'introduction d'une "interdiction de discriminer" qu'à l'ingérence de plus en plus pesante de l'État dans notre système suisse de formation initiale et continue » (Nouvelles, 16 avril 2015). Et de préciser encore que «l'Union patronale met en garde contre toute expérience avec de mauvaises incitations ayant pour effet de renchérir les coûts du travail, de mettre des emplois en danger ou de provoquer un transfert de l'emploi au détriment des actifs plus jeunes » (ibid.). www.arbeitgeber.ch/fr/marche-du-travail/integration-des-travailleurs-ages-la-suisse-dans-le-peloton-de-tete/ 39. Dans un article publié sur le site Internet de la Fédération des entreprises romandes (FER), la question du licenciement abusif - ou non - d'un travailleur âgé a été l'occasion pour son auteur de partir des cas traités au niveau des tribunaux - jurisprudence - pour formuler la recommandation suivante : « II est déconseillé de licencier un employé, proche de l'âge de la retraite, qui travaille depuis de nombreuses années pour l'entreprise et dont les prestations sont satisfaisantes, sans préalablement l'avertir ou sans lui avoir, par exemple, proposé un autre poste. » 
L'analyse du licenciement, telle que perçue par les travailleurs âgés, tend à confirmer cette pratique. En effet, aucune des personnes interrogées n'a déclaré avoir été confrontée à des motifs de licenciement liés à l'âge. Si cela semble attester que les employeurs tiennent compte du cadre légal, une partie des personnes interviewées se fait sa propre opinion, en allant au-delà des raisons formellement notifiées. Ainsi, comment déterminer les véritables raisons du licenciement dans le cas de cet ingénieur manager d'entreprise de 62 ans qui se retrouve «poussé vers la sortie » et remplacé par la propre fille de l'employeur, alors qu'il l'avait lui-même encadrée (lors de stages) durant la période de ses études? Même question dans le cas de cette femme de 63 ans qui ne comprend pas que son entreprise ne lui offre pas un autre poste sous prétexte qu'elle doit quitter son emploi en même temps que son supérieur hiérarchique, lequel a été remercié pour défaut de performances.

Pour expliquer pourquoi ils ne sont pas en activité lorsque nous les avons interviewés, les seniors au chômage évoquent divers motifs qui se combinent avec différents modes de retrait de la vie active (tableau 2). Tout d'abord, il faut distinguer celles et ceux qui ont été licenciés de celles et ceux qui ont démissionné de leur travail. À ces deux modes de départ de l'activité professionnelle s'ajoute le cas des individus qui ne sont pas en activité pour d'autres raisons (retour d'expatriation, demandeurs d'emploi après une expérience non concluante de travailleurs indépendants ou après une période d'invalidité, etc.).

Dans le cas des licenciements, les situations individuelles sont à distinguer des collectives. Au regard des témoignages recueillis, les licenciements individuels constituent le mode de retrait de l'activité professionnelle pour plus de la moitié des personnes; viennent ensuite les démissions, puis les autres formes de retraits (tableau 2). Les licenciements collectifs sont moins observés. Toutefois, cette occurrence plus marquée des licenciements individuels est à analyser à l'intérieur de la catégorie des « 50 ans et plus ». Elle n'exprime pas une tendance globale du chômage de cette catégorie de travailleurs et permet encore moins une comparaison avec d'autres catégories. Chez les femmes, le licenciement individuel représente le mode de retrait prédominant, cependant qu'il y a autant de femmes que d'hommes qui démissionnent. Il en est de même pour les autres modes de retrait : les femmes représentent la moitié des personnes à avoir quitté leur entreprise pour des motifs autres que celui d'un licenciement ou d'une démission.

Concernant les personnes qui ont été licenciées, qui ont démissionné ou qui se trouvent dans une autre situation, leur attitude, leurs motivations et leur désir de retrouver une activité professionnelle sont forts ou faibles en fonction de différentes variables. Chez les unes, la sécurité matérielle prime, en termes de disponibilité de ressources physiques (patrimoine financier, biens immobiliers générateurs de revenus réguliers, vie active du conjoint). Chez les autres, la proximité de la retraite semble diminuer leur motivation à rechercher un emploi rémunéré. C'est particulièrement le cas des individus âgés de 62 ans ou plus. Pour d'autres seniors, moins âgés, les motivations sont fonction des observations et des réflexions qui leur sont faites à l'ORP40 concernant leurs qualifications. À ce niveau, pour ceux qui sont dits faiblement qualifiés - ou devant réorienter leur

40. Peu de « 50 ans et plus » comptent sur les bureaux d’intérim et de placement pour trouver un emploi. Néanmoins, ils y déposent leurs dossiers afin de satisfaire aux injonctions des ORP. 
profil professionnel, les démarches pour retrouver un emploi passeront initialement par des mesures de remotivation, d'apprentissage de la rédaction de $C V$, voire de stages, etc. Les « surqualifiés » sont orientés vers des structures plus adaptées - à l'image du BNF - Programme national de qualification - présentes dans plusieurs cantons francophones et germanophones.

\begin{tabular}{|c|c|c|c|c|c|c|c|}
\hline \multirow{2}{*}{$\begin{array}{l}\text { Typologie des motifs avancés } \\
\text { ou évoqués }\end{array}$} & \multicolumn{3}{|c|}{ Femmes } & \multicolumn{3}{|c|}{ Hommes } & \multirow[t]{2}{*}{ Total } \\
\hline & {$[50-54]$} & [55-59] & {$[60-64]$} & {$[50-54]$} & {$[55-59]$} & [60-64] & \\
\hline Réduction de personnel & 0 & 1 & 1 & 5 & 1 & 1 & 9 \\
\hline Restructuration & 1 & 0 & 1 & 2 & 3 & 0 & 7 \\
\hline Délocalisation & 0 & 1 & 2 & 1 & 3 & 1 & 8 \\
\hline $\begin{array}{l}\text { Tensions sociales/conflits au travail/ } \\
\text { ambiance délétère }\end{array}$ & 2 & 1 & 1 & 1 & 5 & 2 & 12 \\
\hline Difficultés économiques & 1 & 0 & 0 & 1 & 0 & 2 & 4 \\
\hline Faillite/liquidation & 1 & 1 & 2 & 3 & 0 & 2 & 9 \\
\hline Maladie/invalidité & 3 & 0 & 0 & 0 & 0 & 0 & 3 \\
\hline Indisponibilité personnelle & 0 & 2 & 0 & 1 & 2 & 1 & 6 \\
\hline Non-conformité du profil & 0 & 2 & 0 & 2 & 1 & 1 & 6 \\
\hline Contrat non renouvelé & 1 & 0 & 0 & 0 & 0 & 0 & 1 \\
\hline Démotivation & 0 & 0 & 0 & 0 & 0 & 0 & 0 \\
\hline Autre & 0 & 0 & 0 & 0 & 0 & 0 & 0 \\
\hline
\end{tabular}

Source : recherche FNS, « Fin de carrière professionnelle : enjeux suisses d'une situation en voie d'ébullition », 2016.

Comme le montre le tableau 2, les femmes sont plus nombreuses à avoir quitté leur place pour des motifs de maladie et/ou d'invalidité ou encore en raison de l'arrivée du terme de leur contrat sans renouvellement possible. Des motifs de non-conformité du profil ou de délocalisation de l'entreprise ont également été indiqués, ainsi que des situations de faillite de l'entreprise. Les raisons d'ordre économique occupent aussi une place significative dans les motifs de cessation des rapports de travail entre une collaboratrice et son employeur. Les femmes de plus de 60 ans semblent particulièrement exposées à une rupture des rapports de travail lorsque l'entreprise fait face à des difficultés économiques voire lorsqu'elle se trouve en situation de faillite. Nos résultats vont dans le sens de ceux d'une enquête représentative menée en 2003 dans toute la Suisse auprès de personnes ayant pris une retraite anticipée, volontaire ou contrainte. Un tiers des répondants indiquaient des problèmes de santé et un tiers des motifs liés à la situation de l'entreprise (restructuration interne parfois anticipée par les personnes) (OFAS, 2003). Ces mêmes motifs d'ordre économique sont également présents dans les explications fournies par les hommes. S'y ajoute la tendance à davantage démissionner, les motifs se déclinant alors en termes « d'indisponibilité personnelle ». Dans la plupart des cas, il s'agit de personnes qui ont choisi d'aller vers un autre employeur mais dont l'ambition n'a pas abouti, malgré les promesses obtenues. 


\section{En guise d'épilogue}

En Suisse, la régulation publique du marché de l'emploi est peu importante et les travailleurs âgés ne bénéficient pas d'une protection spécifique. Â la lecture des commentaires enregistrés au cours de nos diverses investigations, une confusion collective semble identifiable, mêlant trois dimensions entrelacées, mais pourtant distinguables.

En premier lieu se situe les représentations (voire des stéréotypes) liées à l'âge dans le monde du travail, provenant des collègues comme de l'employeur. Parmi les témoignages recueillis, les observations et réflexions négatives faisant référence à l'âge ont été davantage perçues au cours des tentatives de réintégration que dans la période durant laquelle ces « 50 ans et plus » étaient en activité. Au sein des entreprises, ces représentations se manifestent de manière suffisamment subtile pour ne pas se transformer en tension ouverte entre employeur et employé conduisant à des rapports conflictuels. Par ailleurs, rien n'y est développé pour maintenir et promouvoir les travailleurs âgés, excepté éventuellement les cadres travaillant dans les grands groupes précédemment évoqués. Pour les non-cadres ou les personnes peu qualifiées, l'arrivée au terme d'un contrat de travail constitue un moment d'angoisse, surtout pour les seniors désireux de demeurer en activité, quelle que soit leur motivation. Un accident ou une invalidité attestée expose également certains seniors à une mise à l'écart par l'employeur. Incrédule et dubitatif devant le rôle joué par les représentations de l'âge, réductrices et stéréotypées, un participant à l'un des focus groups s' interrogeait ainsi : «Que signifie "travailleur âgé" pour le président Obama avec ses 56 ans ? Que signifie "travailleur âgé" pour le pape François à plus de 70 ans ? »

En deuxième lieu, des mesures objectivables sont avancées en termes de productivité du collaborateur âgé. Cet argument, essentiellement d'essence patronale, est étayé par des caractéristiques telles que la motivation au travail, la disponibilité, l'absentéisme, la capacité de travail au sens large.

En troisième lieu, il est fait état des limites économiques à l'intégration, voire à la réintégration, des seniors sur le marché du travail. Cette question concerne un cercle plus large de milieux intéressés que les seuls employeurs et employés à commencer par le monde politique. Par exemple, le coût représenté par le senior en termes de salaire ou de charges sociales est régulièrement évoqué comme obstacle à son intégration. À ce sujet, les associations de défense des travailleurs âgés sont convaincues que les caisses de retraite peuvent contribuer à trouver une solution pour atténuer les réticences des employeurs dans le recrutement des seniors.

Ces trois dimensions interagissent entre elles et représentent une sorte d'engrenage duquel le senior peine à s'extraire, alors que c'est sur lui que pèse la responsabilité de trouver une solution pour se maintenir en activité. Le rôle-clé des entreprises en faveur des travailleurs âgés n'est pas suffisamment souligné. C'est probablement le rôle même d'un État libéral que de le rappeler.

La dominante libérale qui préside à l'organisation des rapports de travail en Suisse pénalise particulièrement certaines catégories de seniors en fin de carrière professionnelle. Le chômage, mais aussi l'éviction du marché de l'emploi, touche plus fortement les personnes peu qualifiées qui dépendent alors durablement de la protection sociale. Les pratiques de recrutement des employeurs se révèlent paradoxales, clamant la nécessité de 
maintenir les aînés au travail tout en les écartant par le biais d'une succession de filtres. Les travailleurs âgés sont en compétition avec d'autres groupes, tels que les personnes immigrées qualifiées ou les travailleurs plus jeunes, réputés plus flexibles et entraînant des charges sociales moins lourdes.

Les associations patronales reconnaissent l'importance de maintenir en activité la maind'œuvre âgée, en particulier pour faire face aux difficultés prévisibles de recrutement d'un personnel qualifié, alors même que la marge de manœuvre offerte par l'immigration se réduit. Mais le credo libéral de ces associations empêche les soutiens publics aux seniors, lesquels doivent d'abord compter sur eux-mêmes pour se réintégrer, s'ils perdent leur emploi, et tenir à jour leurs compétences. Ainsi, il incombe aux individus d'être à l'initiative d'une actualisation de leur formation de base par la formation continue et d'en supporter la charge financière.

Parmi les salariés les plus vulnérables se retrouvent d'abord les femmes, pour lesquelles les dispositifs développés ne prévoient aucune aide particulière. Le marché du travail tout comme les dispositifs de prévoyance sociale ont été conçus à partir du modèle traditionnel de participation de l'homme à la vie active avec une carrière à temps plein sans interruption. Ce stéréotype de l'homme qui travaille tout au long de son existence ne représente pas toute la réalité de la vie active. Une partie importante des femmes, actuellement en fin de carrière, ont vu leur vie professionnelle interrompue suite à la naissance d'un ou de plusieurs enfants. Cette vie active est aussi plus souvent accomplie à temps partiel, le revenu des femmes étant alors considéré comme un complément au revenu global du ménage (Rosende, Schoeni, 2012).

Les ruptures dans la carrière professionnelle, les divorces ou les séparations entraînent cette population féminine dans la précarité et la mettent surtout dans l'incapacité de satisfaire complètement aux exigences à remplir pour vivre une retraite professionnelle sans problème matériel. L'absence de mesures spécifiques pour cette catégorie de salariés interpelle tout particulièrement. Au-delà des questions d'égalité, traitées au niveau politique, les femmes de « 50 ans et plus » ne bénéficient d'aucune mesure incitative pouvant favoriser ou encourager un retour en activité. La forte tendance observée dans les statistiques quant à leur retour plus massif sur le marché de l'emploi doit être lue avec prudence. En effet, l'enjeu est complexe en raison de leur poids démographique et de leur plus forte orientation vers des emplois à temps partiel.

L'apport des femmes au marché de l'emploi pourrait être important à l'avenir, surtout dans un pays comme la Suisse en quête de main-d'œuvre qualifiée. Mais cela ne pourra se faire sans, d'une part, une adaptation des conditions générales qui leur seront réservées ou du rôle que l'État leur attribuera et sans, d'autre part, une réflexion fondamentale sur les risques de précarité qui les menacent actuellement et les contraignent à une série de stratégies d'adaptation.

De façon plus générale, au sein des entreprises, l'adaptation des carrières et de leurs trajectoires peut et doit faire l'objet d'une réflexion et d'une planification pour que les qualités qui s'acquièrent avec l'âge trouvent leur place. L'employabilité ne réside pas uniquement dans l'adaptabilité du travailleur au changement ou dans sa capacité à satisfaire les exigences du marché du travail ; elle existe également dans la capacité de l'entreprise à offrir des postes et des tâches mettant en valeur les compétences du senior (Knüsel et al., 2015). 


\section{Bibliographie}

Alberola E., Aldeghi I., Guisse N., 2014, « L'employabilité des demandeurs d'emploi : une approche de la notion et de sa mesure », Crédoc, Cahier de recherche, n C319.

Arni P., 2012, Conseil et coaching intensifs pour demandeurs d'emploi âgés : une voie pour améliorer leurs chances sur le marché du travail ?, Évaluation systématique des résultats, rapport final, Office de l'économie et de l'emploi du canton d'Argovie et université de Lausanne. http://works.bepress.com/patrick_arni/3/

Barbier J.-C., 2002, « Peut-on parler d'activation de la protection sociale en Europe ? », Revue française de sociologie, vol. 43, n², p. 307-332.

Blank S., 2015, « On devrait continuer à se former même à un âge avancé », interview J. N. Schneider-Ammann, La Vie économique, $n^{\circ}$ 7, p. 57-58. https://dievolkswirtschaft.ch/content/uploads/2015/06/22_Dossier_FR.pdf

Bonoli G., et al., 2013, « L'impact des réseaux sociaux sur le retour à l'emploi des chômeurs. Étude de la troisième vague des évaluations de la politique du marché du travail », Étude mandatée par la commission de surveillance du fonds de compensation de l'assurancechômage, Publication du SECO, Politique du marché du travail, $n^{\circ} 37$.

Buchs H., Gnehm A., 2016, Limites d'âge dans les offres d'emploi de 2006 à 2015, Rapport à l'attention du secrétariat d'État à l'économie SECO, Zurich, UNIZH.

Crespo S., 2004, "Les "nouveaux parcours" de fin de carrière », Retraite et société, n 42, p. $65-85$.

Dang A.-T., Zajdela H., 2009, « Fondements normatifs des politiques d'activation : un éclairage à partir des théories de la justice ", Recherches économiques de Louvain, vol. $75, n^{\circ} 3$, p. 313-352.

Dieserens M.-E., Briant H., 1996, Production et reproduction : la femme entre conjoint, enfants et activité professionnelle, en collab. avec Jan Boruvka, Berne, OFS, 260 p.

Gaullier X., 2003, Le temps des retraites. Les mutations de la société salariale, Paris, Seuil (coll. La République des idées).

Gazier B., 1990, «L'employabilité : brève radiographie d'un concept en mutation », Sociologie du travail, $n^{\circ} 4$, octobre-décembre, p. 574-584.

Granovetter M., 1974, Getting a job: a study of contacts and careers, Cambridge Mass, Harvard University, $259 \mathrm{p}$.

Guillemard A.-M., 1993, «Emploi, protection sociale et cycle de vie : résultat d'une comparaison internationale des dispositifs de sortie anticipée d'activité », Sociologie du travail, $n^{\circ} 3$, p. 258-284. 
Guillemard A.-M., 2005, « Politiques publiques et cultures de l'âge : une perspective internationale », Politix, $n^{\circ} 72$, p. 79-98.

Guillemard A.-M., 2013, « Le vieillissement actif : enjeux, obstacles, limites. Une perspective internationale », Retraite et société, $n^{\circ}$ 65, p. 17-38.

ILO - Bureau for Employers' Activities (ACT/EMP), 2015, Femmes d'affaires et femmes cadres : une montée en puissance, Genève, BIT, 44 p.

Jaccard J., 2014, « Licencier un employé proche de l'âge de la retraite est-il abusif ? », Entreprise romande.

https://www.fer-ge.ch/web/fer-ge/-/licencier-un-employe-proche-de-l-age-de-la-retraite-est-il-abusif-

Kite M. E., Johnson B. T., 1988, « Attitudes toward older and young adult: a meta-analysis », Psychology and aging, $\mathrm{n}^{\circ}$ 3, p. 233-244.

Kite M. E., Stockdale G. D., Whitley B. E., Johnson B. T., 2005, « Attitudes toward young and older adults: an updated meta-analytic review », Journal of social issues, $\mathrm{n}^{\circ} 61, \mathrm{p} .241-266$.

Knüsel R., et al., 2015, « Les transformations des politiques de retraite. Introduction au numéro spécial », Revue suisse de sociologie, n 41/3.

Le Goff J.-M., 1999, « Les trajectoires de travailleurs âgés en Suisse et en Europe », Lausanne, Réalités sociales, 97 p.

OCDE, 1998, Maintaining prosperity in an ageing society, Paris, Éditions OCDE, 144 p.

OCDE, 2014, Vieillissement et politiques de l'emploi : Suisse 2014, mieux travailler avec l'âge, Paris, Éditions OCDE, $192 \mathrm{p}$.

http://dx.doi.org/10.1787/9789264222823-fr

OFAS, 2003, « Le passage à la retraite : trajectoires, déterminants et conséquences », Rapport de recherche, $n^{\circ} 2 / 03$, p. 43 et suiv.

OFS, 2008, L'activité des personnes de 50 ans et plus, Vie active et rémunération du travail, n 206-0710, Neuchâtel, OFS, 25 p.

0FS, 2013, Vers l'égalité entre femmes et hommes. Situation et évolution, Situation économique et sociale de la population, $n^{\circ}$ 617-1300, Neuchâtel, 40 p.

www.bfs.admin.ch/bfs/fr/home/statistiques/situation-economique-sociale-population/egalitefemmes-hommes/activite-professionnelle/situation-profession.html

OFS, 2015, «Scénarios de l'évolution de la population de la Suisse 2015-2045. Un vieillissement de la population important ces trente prochaines années », Communiqué de presse, Neuchâtel, OFS.

Posthuma R.-A., Campion M.-A., 2007, « Age stereotypes in the workplace: common stereotypes, moderators, and future research directions », Journal of management, vol. 35, p. 158-188. 
Vieillir en entreprise : logiques organisationnelles et genre

Rosende M., Schoeni C., 2012, " Seconde partie de carrière, régime de retraite et inégalités de sexe, le cas suisse ", Revue française des affaires sociales, n² 2-3, p. 130-147.

SEC0, 2016, « Indicateurs de la situation des travailleuses et travailleurs âgés sur le marché suisse du travail », Documents de base pour la conférence nationale du 21 avril 2016, Berne, $25 \mathrm{p}$.

SEC0, 2017, Chômage des personnes âgées de 50 ans et plus (50+), Rapport, Berne, 24 p.

Tschumi M., 2016, Enquête réalisée en collaboration avec l'émission On en parle - RTS La Première, diffusée le 3 novembre.

www.generations-plus.ch

Turtschi N., 2015, Les réseaux sociaux, un outil de réinsertion pour les chômeurs désavantagés, Thèse en administration publique, université de Lausanne, 200 p. urn:nbn:ch:serval-BIB_D548639B4D090

Vogt V., 2015, «Les salariés doivent être ouverts au changement », La Vie économique, $n^{\circ} 7$, p. 60.

https://dievolkswirtschaft.ch/content/uploads/2015/06/22_Dossier_FR.pdf

Weber B., 2015, « La situation des travailleurs âgés est bonne », La Vie économique, $\mathrm{n}^{\circ} 7$, p. 54-56.

https://dievolkswirtschaft.ch/content/uploads/2015/06/22_Dossier_FR.pdf

Widmer E., Ritschard G., 2009, « The de-standardization of the life course: are men and women equal? », Advances in Life Course Research, vol. 14, n 1-2, p. 28-39. 